\title{
An Element in a Paradigm Shift? - Could Population II Stars Actually be Younger than Population I stars?
}

\author{
Arne Bergstrom* \\ B\&E Scientific Ltd, BN25 4PA, United Kingdom \\ *Corresponding author: arne.bergstrom@physics.org \\ Received May 01, 2014; Revised May 30, 2014; Accepted June 04, 2014
}

\begin{abstract}
Recent astronomical observations have indicated the existence of supermassive black holes at the centres of most, perhaps all, galaxies, and also that immensely powerful relativistic jets of protons and electrons may occasionally emanate from some such supermassive black holes. Together these observations suggest a possible mechanism for alternative star formation, which may require a revision of the traditional classification of stars into a more recent Population I and a more ancient Population II. Rather than assumedly being members of an older generation of stars preceding Population I, maybe many Population II stars could perhaps actually be younger than Population I stars, with their lower metallicity being an effect of their much more recent creation from the pristine hydrogen left by such relativistic jets.
\end{abstract}

Keywords: Big-bang vs steady-state, supermassive black holes, relativistic jets

Cite This Article: Arne Bergstrom, "An Element in a Paradigm Shift? - Could Population II Stars Actually be Younger than Population I stars?” International Journal of Physics, vol. 2, no. 3 (2014): 78-82. doi: 10.12691/ijp-2-3-2.

\section{Introduction}

According to Kuhn [1], scientific progress may often occur via paradigm shifts, i $e$ drastic, revolutionary changes in our view of certain problems. The big-bang theory of the evolution of the universe has been used as an example of such a paradigm shift away from a steady-state model of the universe. However, based on a revised view of certain observational facts, the present communication discusses whether there might possibly be a case for a tentative paradigm shift back to a kind of modified steadystate model again.

As an element suggesting such a revised view, it has been shown that the cosmological redshift need not necessarily be an effect of an expanding universe at all, but could:

(1) be an observational effect [2] due to the requirements of relativistically invariant quantum propagation through space while at the same time obeying the exact Boltzmann transport equation.

If so, this can then also be seen [3] to explain:

(2) why the expansion of the universe appears accelerating, and

(3) why there appears to be a dominating background of dark energy in the universe.

Rather than as an afterglow from a primordial universe, it is then also possible to:

(4) interpret the exact blackbody spectrum of the cosmic microwave background as an effect [4] of such an apparent, exponentially accelerated expansion of the universe.
According to this approach, dark energy and the accelerated expansion would thus be independent consequences of the requirements of a Lorentz-covariant quantum propagation, and hence are not dynamically connected to each other. The accelerated expansion is not driven by any pressure from the dark energy. This thus eliminates the crucial problem of how to otherwise understand the huge discrepancy by between about sixty to more than a hundred orders of magnitude in this connection [5], and which has been called "the worst theoretical prediction in the history of physics".

\section{A remaining Question}

If there is indeed a valid alternative picture of the kind outlined above, then a most important question would still remain. This is the question why the abundances of naturally occurring radioactive elements, such as uranium and thorium, seem to indicate an age from when they were created that agrees in order of magnitude with the assumed estimated age of the universe as determined from the cosmological redshift.

One possible alternative way to approach this question would then be to consider - as will be done below - the galaxies themselves to be the factories of the elements from which they are made. We could then think of a life cycle of these elements, in which they are cyclically being drawn into a supermassive black hole in the centre of the galaxy, and then intermittently re-emitted as narrow relativistic jets of pristine hydrogen. From these jets, star clusters are then formed containing newly born metal-poor stars. Eventually the stars in these clusters are then drawn 
towards the galactic disk, and from there they then eventually migrate towards the centre of the galaxy. In this process, nucleosynthesis and supernova explosions produce the heavier elements, and these second or later generations of stars are then finally eventually drawn back into the central black hole to be rejuvenated there, and the cycle starts all over again, etc.

If such a continuous cycle would take the order of ten billion years to complete, then this would be a way to understand the evolution of the universe in a kind of local steady-state scenario. The cosmological evolution would according to this scenario then essentially take place within each galaxy (with some exchange of material between neighbouring galaxies in connection with the relativistic jets). The apparent age of a galaxy would then depend on how long this regeneration cycle would take in each individual galactic group.

With regard to the ages of radioactive elements, this scenario would thus not in any substantial way differ from a big-bang model. In the following, this alternative scenario will be discussed in somewhat more detail, although the presentation of an alternative to the extensively studied big-bang model must with necessity in comparison be somewhat sketchy. The reason for discussing it at all at this stage is to outline an alternative cosmological model, which as discussed above agrees with the big-bang model in a number of ways, and which could be used as a means to critically test conclusions from the big-bang model against a possible alternative scenario.

\section{Could Population II Actually be Younger than Population I?}

Stars in Population II, which essentially form a halo around the core of our Milky Way galaxy (see Figure 1), are traditionally considered to be older than the Population I stars forming the disk in our galaxy [7]. This conclusion is based on the observation that Population II stars have substantially lower metallicity than Population I, $i$ e they contain substantially less of the heavier elements that could only have been formed in stars at a later stage after the assumed initial big bang (by convention such heavier elements are summarised as "metals"). Instead, such heavier elements found in Population I stars are assumed to require supernova explosions for their creation, and thus require the existence of a previous generation of stars as the sources for such supernovae. Hence it is assumed that stars belonging to Population I were preceded by a generation of stars in which such heavier elements were produced when the stars in this earlier generation subsequently underwent supernova explosions. Population II with its lower metallicity was first considered as a possible candidate for such a previous generation of stars.

However, a problem with this scenario is that Population II stars, although metal-poor, still contain small amounts of heavier elements, which these stars must have picked up from an interstellar medium during their formation. Thus Population II stars cannot be the truly primeval stars, $i$ e they cannot be the very first stars born after the assumed big bang. Such truly primeval stars are instead believed to belong to a hypothetical Population III
[8] of still earlier stars containing no heavier elements at all (see Figure 2).

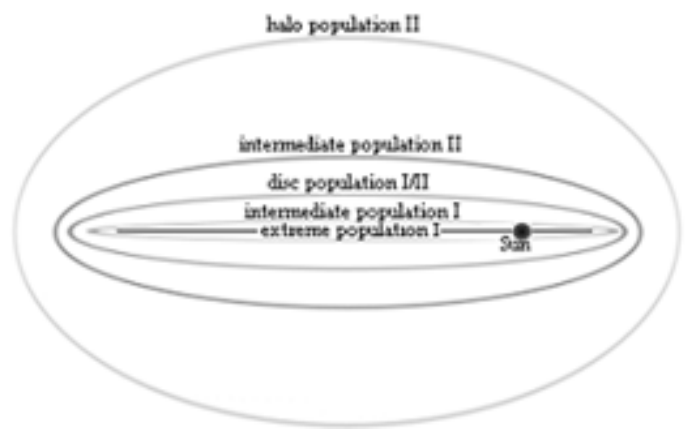

Figure 1. Distribution [6] of star populations in the Milky Way, showing how Population II stars essentially form a halo around the disk of the Milky Way where the stars in Population I are concentrated

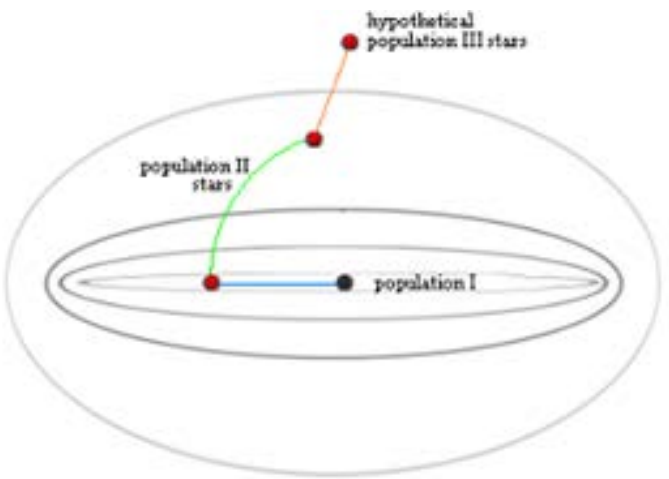

Figure 2. Assumed creation of stars. The first stars start as hypothetical, metal-free Population III stars after the big bang, which then within a few hundred million years become supernovae and seed the interstellar space with sufficient heavy elements to produce Population II stars. These stars then in turn become supernovae and finally produce the material for Population I stars, which eventually may form a supermassive black hole in the galactic centre

A problem here, however, is that no such Population III stars have ever been observed. If they existed at all at some earlier stage in the evolution of our galaxy, then there must have been some mechanism causing them all to go extinct within a few hundred million years after their creation from material from the assumed big bang (which in fact may be quite possible due to their special composition).

However, a more serious problem with the traditional interpretation outlined above of Population II as being older than Population I, is that Population II stars are mainly found in a halo around the disk of Population I stars in our galaxy. It is then not clear how the assumedly older Population II stars can have avoided the general mechanism due to gravitational forces that have caused all the orbits of all the then assumedly younger stars in Population I to develop into a thin disk.

One possibility is that the Population II stars - like the comets in our own solar system - belong to a population of old objects with very elongated orbits that keep them out of the gravitational influence of the more central objects for a considerable proportion of their orbits. However, Population II stars do not seem in general to have the very elongated orbits required, so this may not be a likely explanation for their distribution.

Another possibility is that the creation of Population II stars took place outside our galaxy, and that they have then more recently become captured by the gravitational 
attraction from it. But if the Population II stars are old, then one would still expect a majority of them to have been subjected to the gravitational forces in our galaxy earlier on, and thus still to have ended up in the disk just as the Population I stars. This is not the case - the Population II stars seem to be found in the galactic disk essentially only to the extent that their rotation around the galactic centre is expected to take them there.

\section{Central Supermassive Black Hole}

From the above arguments, one would be tempted to assume that most Population II stars discussed above might actually be young members of the stars in the Milky Way galaxy, and maybe in fact be younger than Population I stars.

However, this explanation would then immediately raise the very serious objection of how the then assumedly younger stars in Population II can possibly have the more primitive composition they have with much lower metallicity than the then assumedly older stars in Population I.

If Population II stars were born much more recently than Population I stars, then one would instead expect them during their formation to have had access only to material of the same or higher metallicity than the stars in Population I, not the material of considerably much lower metallicity that they have obviously all been formed from.

This question thus becomes intimately coupled to the question of where and how Population II stars were created. We now know that most galaxies, possibly all, have in their centre a supermassive black hole [9]. Such a supermassive black hole attracts stars and eventually devours them when they come too close. All detailed characteristics concerning, $\mathrm{eg}$, element composition of the captured star is then lost; it enters the supermassive black hole only as an essentially anonymous contribution to the mass/energy and angular momentum of the supermassive black hole.

It is here assumed that such capture processes might occasionally lead to a temporary instability in the supermassive black hole, which it tries to correct by one of the very few ways open to it - the emission of two narrow, extremely powerful relativistic jets emitted from its poles, as have been observed in some galaxies [10]. These relativistic jets are assumed to consist of electrons, positrons, and protons, and are emitted with velocities very close to the speed of light. They can reach thousands or even hundreds of thousands of light-years from their parent galaxy, $i$ e comparable to the extension of the galaxy itself. No such jets are at the present observed from the supermassive black hole in our own galaxy, but may conceivably have happened very many times previously during its existence.

A relativistic jet as discussed here (a proton beam with accompanying electrons to keep it quasi-neutral) is expected - as charged particle beams do - to have a certain well-defined range depending on its proton energy, and thus determining where the particles will finally be thermalized and deposited. As is typical for a chargedparticle beam, there will be very few losses of particles along the way before it reaches its range. This thus means that when thermalised, the particles in the assumed narrow relativistic jets emitted from a super-massive black hole are expected to accumulate in a very small region at the end of this range, and do so for as long as the relativistic jet remains active. It seems reasonable to assume that the thus accumulated material from such a relativistic jet may coalesce to form localised clusters of stars, which would then have the same age and be held together by their mutual gravitational attraction.

\section{Globular Clusters}

It is tempting to assume that the clusters of stars we see in the form of globular clusters as satellites to the Milky Way [11] could perhaps be the remnants of earlier such relativistic jets in our galaxy (or occasionally maybe even from neighbouring galaxies). The relativistic jets, from which the clusters in this scenario were formed, occur in pairs in opposite directions. Hence, the clusters are expected to appear in pairs, one on each side of the galactic plane (although each such cluster would subsequently drift away independently due to their respective motions in the galaxy).

It should be pointed out that the view presented here of a comparatively recent age of globular clusters is in stark disagreement with the general view, which suggests the age of globular clusters to be more like the age recently estimated for the Population II star HE 1523-0901 as discussed in section 6 below.

On the other hand, the assumption made here of relativistic jets relieving instabilities in supermassive black holes, and in this way producing globular clusters, could perhaps find support in the surprising correlation recently found [12] in a number of galaxies between the mass of their supermassive black holes and the corresponding total mass of their globular clusters. This could indicate a mechanism like the one proposed in this paper, in which old Population I stars from the galactic disk after being drawn into the central black hole are rejuvenated in the form of pristine hydrogen and fed through the relativistic jets to produce new Population II stars in the form of globular clusters in the galactic halo (see Figure 3). Such a mechanism would be expected to create a correlation between the mass of the central supermassive black hole and the total mass of the globular clusters created.

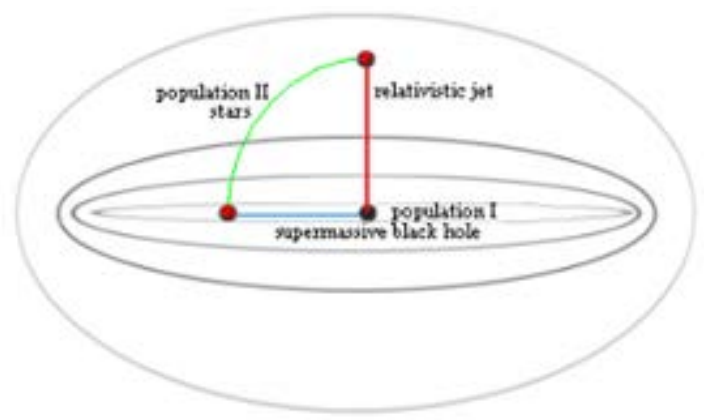

Figure 3. Possible post-population-III creation of stars. Relativistic proton jets from the supermassive black hole in the galactic centre are thermalized and form globular clusters of Population II stars, the material of which after supernova explosions ends up as Population I stars in the galactic disc, and then eventually gets drawn back into the central black hole in the galactic centre, from which it again may be emitted as pristine hydrogen in the form of relativistic jets, etc 
Relativistic jets as discussed above are thus here assumed eventually to form interstellar clouds of hydrogen, which could then contract into stars. This would then be a quite different mechanism for star formation than the one occurring with the Population I stars in the galactic plane, where they are formed from interstellar material, including material from supernova explosions of older Population I stars, thus giving rise to their higher metallicity. In contrast, Population II stars from relativistic jets as discussed here would in principle have zero metallicity. However, they would be expected to contain some elements from the interstellar medium in which they are formed, $i$ e they would be expected to have a certain - but low - metallicity.

\section{A Paradox about Age}

From the discussion above follows that there may possibly exist two types of Population II stars of radically different age - one population of very ancient stars and one population of very recent stars. It is not obvious if these two types of Population II stars have any relation to the two types of Population II stars that have been discussed previously in the literature $[13,14]$.

In this connection a possible, and somewhat paradoxical problem related to estimating the age of Population II stars needs to be pointed out. At least some such stars are here assumed to have received their limited metallicity from the interstellar/intergalactic medium, in which the relativistic jet got thermalised and coalesced into stars. In a prominent study [15], the age of such a Population II star, namely HE 1523-0901, has been determined by measuring its abundance of uranium and thorium relative to stable heavy nuclides.

However, this approach risks estimating only the age of the polluting interstellar medium that the hydrogen jet has picked up when thermalising and coalescing into stars as discussed above. The age of the star estimated in this way from its interstellar pollution may then easily give values close to the assumed age of the universe, despite the fact that the star itself may perhaps only be a very small fraction of the age estimated in this way.

Thus determining the age of Population II stars by analysing their abundance of heavier isotopes will not distinguish between the two possible age categories of Population II stars discussed here. In order to estimate the age of such a Population II star reliably, one might be forced to try to estimate its age from more pristine nuclides like lithium and beryllium, after first deducting the estimated contribution from interstellar-medium pollution as discussed above. It is noteworthy in this connection that there seems to be a discrepancy [16] in the observed lithium abundance compared to what would be expected, which might indicate that considerations as discussed here perhaps need to be taken into account.

It is thus here assumed that the stars produced in the way discussed above are gravitationally attracted to their parent galaxy (or possibly to a neighbouring galaxy) in the form of Population II stars. As a result of the mechanism described here, these Population II stars would then be younger than their Population I cousins, but compared to which they would nevertheless have much lower metallicity, as is indeed observed. A Population II star with a seemingly ancient isotope composition may thus, as suggested above, not necessarily be an old fossil at all.

\section{Conclusion}

It is in this paper proposed that at least some Population II stars may actually be younger than the Population I stars we see in the Milky Way today, and to have required supermassive black holes and relativistic jets for their creation, as described above.

Assuming a big-bang scenario, we must envisage a Population III of hypothetical primeval, metal-free stars as discussed in the beginning of this paper. In this scenario, these Population III stars must then be assumed to have been the very first stars in our galaxy after the assumed big bang (cf Figure 2). These very first stars are then assumed to have produced the first heavier elements through supernova explosions, which then successively have given rise to the intergalactic medium and the Population I stars with the metallicity we have today, including some Population II stars along the way. As mentioned above, the fact that no Population III stars have been observed is ascribed to the reasonable assumption that they were short-lived (less than some hundred million years) as a result of their primitive composition. Similarly, due to their composition Population II stars would also be expected to be more massive and move faster through the Hertzsprung-Russel diagram than Population I stars.

Contrary to common perception, it could perhaps be that most Population II stars we see today were not formed until Population I stars had existed for sufficient time to form the supermassive black holes and relativistic jets considered here to be the main birthplace of the Population II stars. Their more recent formation would then explain the distribution of Population II stars predominantly in the galactic halo, as illustrated in Figure 1. This scenario might even lead to a repetitive life cycle of stars as discussed in the Introduction, and which in our present epoch no longer contains any Population III stars, as illustrated in Figure 3. New efforts are scheduled for the very near future to find the missing Population III stars. Should against all expectations these efforts turn out to be unsuccessful, then scenarios like what is discussed above with respect to Figure 3 above may perhaps need to be considered.

\section{References}

[1] T. Kuhn, The Structure of Scientific Revolutions (Kindle edition, 2012).

[2] A. Bergstrom, "Relativistic invariance and the expansion of the universe”, Nuovo Cimento 27B, 145 (1975).

[3] A. Bergstrom, "Lorentz-covariant quantum transport and the origin of dark energy”, Phys. Scr. 83, 055901 (2011).

[4] A. Bergstrom, "Is CMB just an observational effect of a universe in accelerated expansion?”, International Journal of Physics, Vol. 1, No. 6, 133-137 (2013).

[5] J. Frieman, M. Turner, and D. Huterer, "Dark Energy and the Accelerating Universe”, Ann. Rev. Astron. Astrophys. 46385 (2008).

See also en.wikipedia.org/wiki/Cosmological_constant.

[6] G. Larsson-Leander, Astronomi och Astrofysik, $2^{\text {nd }}$ Ed (Liber, Stockholm, 1977).

[7] B. Carr, "The cosmic microwave background and particle physics”, Ann. Rev. Astr. Astroph. 32531 (1994). 
[8] V. Bromm \& R. Larson, “The first stars”, Ann. Rev. Astr. Astroph. 4279 (2004).

[9] R. Schödel, T. Ott, R. Genzel, R. Hoffmann, et al, "A star in a 15.2-year orbit around the supermassive black hole at the centre of the Milky Way“, Nature 419694 (2002).

[10] A. Wehrle, et al, https://science.nrao.edu/science/ Decadal\%20Survey/rac/Wehrle_AGN_jets_GCT.pdf/view, retrieved 2012-05-23.

[11] R. Powell, http://www.atlasoftheuniverse.com/globular.html, retrieved 201205-28.
[12] A. Burkert \& S. Tremaine, "A correlation between central supermassive black holes and the globular cluster systems of early-type galaxies”, Astroph. J. 720516 (2010).

[13] H. Decauwer, E. Jehin, G. Parmentier, P. Magain, “Abundance correlations in mildly metal-poor stars”, Astron. \& Astroph. 433 285 (2005).

[14] E. Jehin, P. Magain, C. Neuforge, et al, "Field halo stars: the globular cluster connection”, Astron. \& Astroph. 330 L33 (1998).

[15] A. Frebel, et al, "Discovery of HE-1523-0901, a strongly r-process enhanced metal-poor star with detected uranium”, Astroph. J. 660 L117 (2007).

[16] Fields, B. D., “The Primordial Lithium Problem”, Ann. Rev. Nucl. \& Particle Sc. 61, 47 (2011) 